\title{
PERFORMANCE OF PARAMETER-DOMAIN AND SPATIAL-DOMAIN POLE-LIKE FEATURE SEGMENTATION USING SINGLE AND MULTIPLE TERRESTRIAL LASER SCANS
}

\author{
Z. Lari, K. Al-Durgham, A. Habib \\ Dept. of Geomatics Engineering, University of Calgary, 2500 University Drive NW, Calgary, Canada, T2N1N4 \\ (zlari, kmaldurg, ahabib)@ucalgary.ca
}

Commission V, WG III/3

KEY WORDS: Terrestrial Laser Scanning, Segmentation, Registration, Parameter-domain, Spatial-domain

\begin{abstract}
:
Terrestrial laser scanning (TLS) systems have been established as a leading tool for the acquisition of high density three-dimensional point clouds from physical objects. The collected point clouds by these systems can be utilized for a wide spectrum of object extraction, modelling, and monitoring applications. Pole-like features are among the most important objects that can be extracted from TLS data especially those acquired in urban areas and industrial sites. However, these features cannot be completely extracted and modelled using a single TLS scan due to significant local point density variations and occlusions caused by the other objects. Therefore, multiple TLS scans from different perspectives should be integrated through a registration procedure to provide a complete coverage of the pole-like features in a scene. To date, different segmentation approaches have been proposed for the extraction of pole-like features from either single or multiple-registered TLS scans. These approaches do not consider the internal characteristics of a TLS point cloud (local point density variations and noise level in data) and usually suffer from computational inefficiency. To overcome these problems, two recently-developed PCA-based parameter-domain and spatial-domain approaches for the segmentation of pole-like features are introduced, in this paper. Moreover, the performance of the proposed segmentation approaches for the extraction of pole-like features from a single or multiple-registered TLS scans is investigated in this paper. The alignment of the utilized TLS scans is implemented using an Iterative Closest Projected Point (ICPP) registration procedure. Qualitative and quantitative evaluation of the extracted pole-like features from single and multiple-registered TLS scans, using both of the proposed segmentation approaches, is conducted to verify the extraction of more complete pole-like features using multipleregistered TLS scans.
\end{abstract}

\section{INTRODUCTION}

Over the past few years, Terrestrial Laser Scanning (TLS) systems - static or mobile - have been proven as an efficient and reliable technology for the acquisition of high density point clouds over physical surfaces. The collected point clouds can be utilized for the extraction, modelling, and monitoring of different objects, e.g., building facades, road surfaces, corridors, road furniture, and curbs. The original TLS point cloud usually does not exhibit the semantic information about the type and characteristics of the scanned surfaces. Hence, the collected TLS data should be processed to provide the required information for the aforementioned modelling and monitoring applications. Pole-like features (traffic lights, street lights, utility poles, and road signs) are amongst the most important objects that can be encountered and detected in TLS point clouds, especially those collected in urban areas and industrial sites. A complete extraction and 3D modelling of pole-like features through the processing of a single TLS scan is usually not feasible due to significant variations in the local point density, scanning angle, or occlusions caused by other objects. Consequently, the integration of multiple TLS scans from different perspectives is essential to ensure a complete coverage of the pole-like features. This integration is carried out through a registration procedure which aims at the alignment of different TLS scans to a common reference frame.
In order to extract pole-like features from single or multipleregistered TLS scans, different spatial-domain, parameterdomain and hybrid techniques have been introduced so far. The spatial-domain techniques are mainly classified into regiongrowing based techniques (Belton and Lichti, 2006; Gross and Thoennessen, 2006; El-Halawany and Lichti, 2011) and modelfitting methods (Lukács et al., 1998; Marshall et al., 2001). In region growing-based approaches, a Principal Component Analysis (PCA) of the local neighbourhood of individual laser points is initially implemented for the classification of the points which belong to pole-like features. A region growing approach is then performed while utilizing arbitrary seed points to aggregate neighbouring points which belong to linear/cylindrical features. Although these approaches can reliably and efficiently extract pole-like features, their outcome is highly sensitive to the size of the locally established neighbourhoods for PCA and the choice of seed points. The second category of spatial-domain techniques, model-fitting methods, is implemented in two steps. In the first step, a segmentation approach is used to detect different surfaces (planes, pole-like features, spheres, and cones) in TLS data and select appropriate models for their representation. In the second step, a non-linear least squares procedure is utilized to define best-fitted cylinders to the segmented pole-like surfaces. The drawback of these methods is that the quality of the segmented pole-like features is highly dependent on the quality of initial 
values of their representing parameters that are estimated using non-linear least squares procedure.

In the parameter-domain techniques, the geometric attributes representing pole-like features are initially estimated - seven (Lukács et al., 1998) or five (Rabbani, 2006) directional, positional, and radius parameters. A seven/five-dimensional attribute space is then established for clustering the individual pole-like features. The key drawback of these methods is their computational inefficiency when dealing with a massive amount of points. In order to optimize the computational efficiency of this clustering procedure, the pole-like features are identified in directional and positional/radius attribute subspaces, respectively (Kimme et al., 1975; Rabbani and van den Heuvel, 2005; Rabbani, 2006; Vosselman et al., 2004). For the pole-like features represented by five parameters, a Gaussian sphere of the point cloud is initially constructed and a 2D Hough transform is performed to identify the features which have similar axis direction in the established Gaussian sphere. A 3D Hough transform is then carried out to identify these features based on their position and radius parameters. This sequential clustering procedure greatly improves the efficiency of parameter-domain segmentation of pole-like features. The other limitation of these approaches is that they are not able to discriminate spatially-disconnected coaxial pole-like features. In existing hybrid approaches, pole-like features are initially classified in the parameter domain and then precisely modelled using a least-squares cylinder fitting procedure in the spatial domain (Bolles and Fischler, 1981; Chaperon and Goulette, 2001; Fischler and Bolles, 1981; Schnabel et al., 2007). The drawback of these approaches is that they cannot reliably separate coaxial pole-like features with different radii in the spatial domain.

In order to overcome the limitations of the aforementioned techniques, this paper presents two recently-developed PCAbased parameter-domain and spatial-domain approaches for the segmentation and extraction of pole-like features from TLS scans. These approaches are developed while considering the internal characteristics of the TLS scans. Furthermore, the performance of the proposed approaches for the segmentation of pole-like features using a single or multiple TLS scans is investigated in this paper. The alignment of multiple TLS scans to a common reference frame is performed using an Iterative Closest Projected Point (ICPP) registration approach (AlDurgham et al., 2011) which will be discussed later in this paper.

This paper starts with the introduction of the proposed spatialdomain and parameter-domain approaches for the segmentation and extraction of pole-like features from TLS scans. In the next section, the utilized approach for the registration of multiple TLS scans is briefly reviewed. The performance of the proposed approaches for the segmentation of pole-like features from single or multiple-registered TLS scans is then assessed through qualitative and quantitative evaluation of experimental results using real datasets. Finally, concluding remarks and recommendations for future research work are presented.

\section{POLE-LIKE FEATURE SEGMENTATION AND EXTRACTION}

In this section, the proposed approaches for the segmentation of pole-like features from TLS point clouds are introduced and discussed, in detail. These methods try to overcome the drawbacks of the abovementioned segmentation techniques by considering the local point density variations, geometric characteristics of associated pole-like surfaces (directional and positional parameters), and noise level within the TLS point clouds (either single or multiple-registered scans). Figure 1 illustrates the main steps of the proposed segmentation approaches. The detailed explanation of these steps is presented in the following subsection.

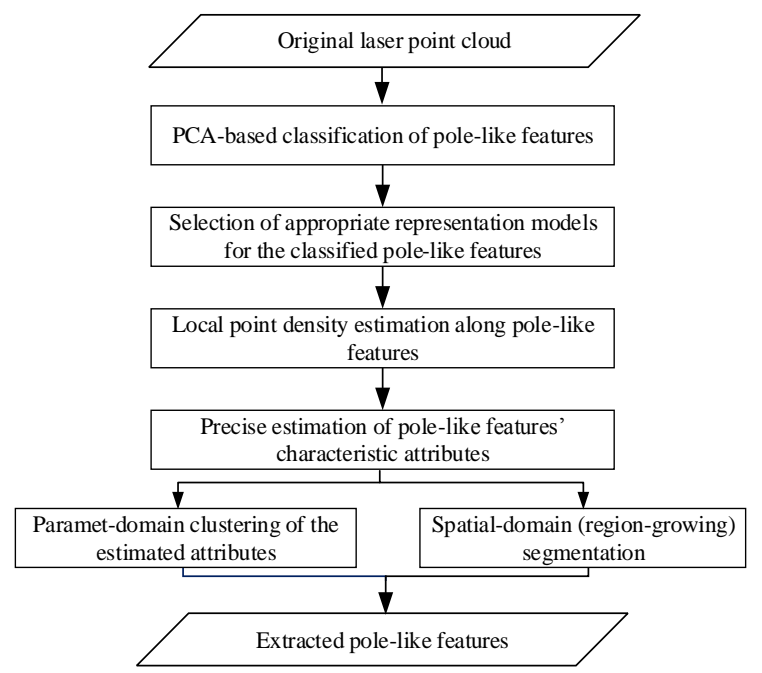

Figure 1.The outline of the proposed methodologies for the segmentation of pole-like features

\subsection{Classification and representation of pole-like features}

In order to handle a massive amount of points in TLS data more efficiently, some level of organization should be introduced to this data before further manipulation steps. Therefore, the kdtree data structure is established for organizing the point cloud (Friedman et al., 1977). The main benefit of this structure is faster and more efficient search and retrieval of the nearest neighbouring points which will be utilized in further data processing activities. Once the laser points are organized, a PCA of their local 3D neighbourhoods is performed (Pauly et al., 2002). Since the pole-like features appear as linear/cylindrical objects in TLS data, the local neighbourhoods are established to enclose the required number of the points for reliable line/cylinder definition. The nature of the point cloud within the established 3D neighbourhood (planarity, linearity, or roughness) is then investigated through eigenvalue analysis of the dispersion matrix of the neighbouring points relative to their centroid. For the local neighbourhoods enclosing a linear/cylindrical feature, one of the normalized eigenvalues will be distinctively larger when compared to the other two (Figure 2). The orientation of the eigenvector corresponding to the largest eigenvalue (pink arrow) represents the approximate direction of the detected pole-like feature.

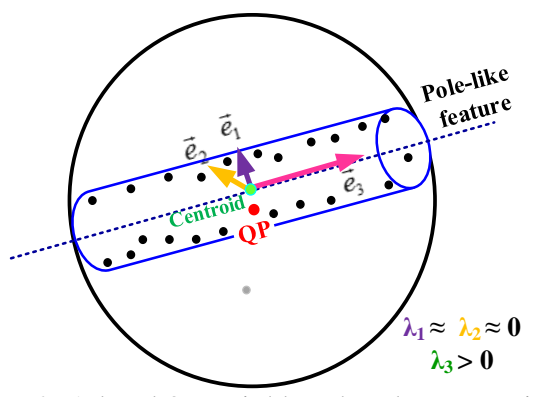

Figure 2. A local 3D neighbourhood representing a pole-like feature

Once the pole-like features are classified using the PCA procedure, appropriate representation models should be defined to describe their characteristic attributes. The axis of a typical pole-like feature is usually represented by six parameters (three 
directional and three positional parameters) in 3D space. The approximate direction of the pole-like features' axes, which is estimated through the PCA, is considered as the basis for the selection of their appropriate representation form. In order to avoid singularities in pole-like features' representation and decrease the number of the required parameters for their segmentation, three representation models are introduced in this research to describe each of these features in the spatial domain. Figure 3 shows three instances of pole-like features whose axes are modelled using the proposed representation forms. One should note that the selection of these models reduces the number of required characteristic attributes for the representation of pole-like features' axes from six (three directional and three positional) to four (two directional and two positional).

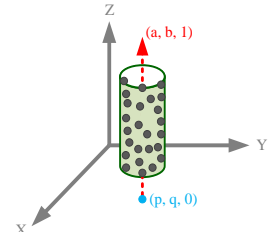

Pole type 1:

$\frac{X-p}{a}=\frac{Y-q}{b}=Z$

Figure 3. Instances of pole-like features represented using the proposed models

\subsection{Local point density estimation}

The local point density is one of the most important internal characteristics of TLS data which should be considered during various processing activities. The effective quantification and consideration of these indices greatly improves the quality of the segmentation and feature extraction from TLS point clouds (Lari and Habib, 2013). For the points which belong to pole-like features, these indices should be estimated while considering the physical properties of their enclosing surfaces. Hence, a cylindrical buffer surrounding a pole-like feature is established to precisely estimate its characteristic parameters and quantify the local point density variations on its surface (Figure 4).

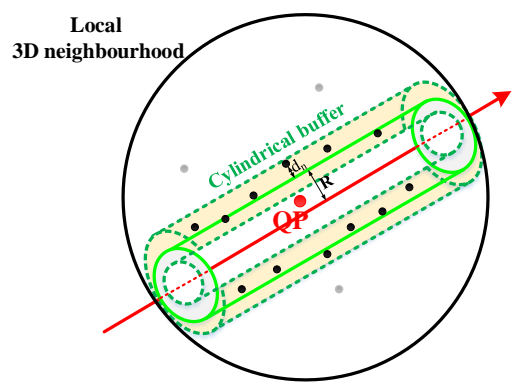

Figure 4. Cylindrical buffer established for local point density estimation along pole-like features

\section{This cylindrical buffer is defined as follows:}

- The height of this cylindrical buffer is specified by the distance between the query point and its $n^{\text {th }}$-furthest neighbour, where $n$ is the required number of points for reliable cylinder definition;

- The direction of this cylindrical buffer is derived through an iterative cylinder fitting procedure which aims at minimizing the squared sum of the normal distances between the points within a local 3D neighbourhood and the surface of the pole-like feature in question; and

- The buffer size surrounding a pole-like feature - the maximum admissible normal distance between a neighbouring point and the surface of pole-like feature - is determined while considering the noise level in the point cloud.

The local point density indices, for the points which have been classified as a part of a pole-like feature, are then evaluated according to Equation 1.

$$
L P D\left(p t s / m^{2}\right)=\frac{k}{4 \pi R r_{n}} \text { in which } \quad k<n
$$

Where $k$ is the number of the points within the adaptive cylindrical buffer; $r_{n}$ is the distance between the query point and its $n^{\text {th }}$-furthest neighbour within the established 3D neighbourhood; and $R$ is the radius of the cylindrical feature estimated through the iterative cylinder fitting procedure (Figure 4). It should be noted that the proposed approach only considers the points within the adaptive cylindrical buffer for local point density computation. Therefore, it is considered as a convenient method for the evaluation of local point density variations along the pole-like features. Once these local point density indices are estimated for the points belonging to polelike features, the PCA-based point classification is repeated while considering these indices for the establishment of 3D neighbourhoods of the query points. This will lead to more precise estimation of the parameters representing pole-like features.

\subsection{Parameter-domain segmentation of pole-like features}

Once the detected pole-like features are precisely represented using directional and positional parameters - while considering the estimated local point density indices, a recently-developed parameter-domain approach is implemented for the segmentation and extraction of these features from TLS data. The objective of this method is to optimize the computational efficiency of the parameter-domain segmentation procedure when dealing with high-dimensional attribute spaces. This optimization is implemented through following steps:

- The cluster detection procedure is carried out in lowerdimensional manageable attribute subspaces (i.e., separate two-dimensional directional and positional attribute subspaces for the pole-like features modelled by each representation form), sequentially;

- The peak detection is performed without the need for having a tessellated accumulator array; and

- The extent of clusters in the attribute subspaces is meaningfully defined.

In this approach, the estimated directional and positional attributes - for the pole-like features modelled by each representation form - are organized in two kd-tree data structures which are manipulated for cluster detection. The appropriate cluster extent for each attribute point in the directional and positional attribute subspaces is then estimated while considering the admissible angular deviation among almost parallel and minimum acceptable distance between almost coaxial pole-like features. The proposed sequential clustering procedure is initiated in a two-dimensional directional attribute subspace. A brute-force peak detection approach (Lari and Habib, 2014) is then carried out to find clusters in directional attribute subspace, while considering the estimated clusters' extent (Figure 5). For each of the detected clusters of attribute points in the directional attribute subspace (representing parallel pole-like features in the spatial domain), the clusters of attributes in the positional kd-tree structure are then identified to discriminate and extract individual pole-like features (Figure 6). 


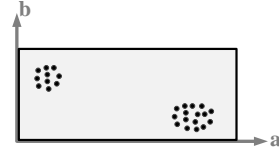

(a)

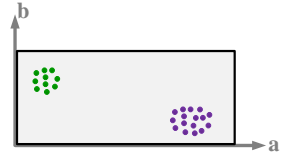

(b)

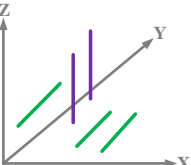

(c)
Figure 5. Clustering procedure in the directional attribute subspace: (a) 2D directional attribute subspace, (b) detected peaks in the directional attribute subspace, and (c) parallel lines representing the detected peaks in the spatial domain

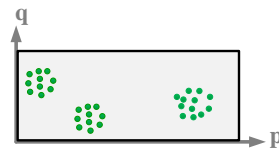

(a)

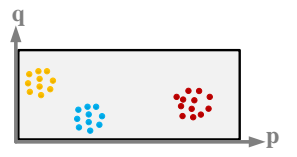

(b)

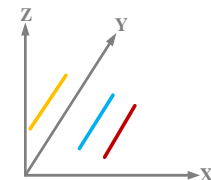

(c)
Figure 6. Clustering individual pole-like features in the positional attribute subspace: (a) $2 \mathrm{D}$ positional attribute subspace, (b) detected peaks in the positional attribute subspace, and (c) extracted lines representing detected peaks in the spatial domain

The main drawback of the parameter-domain methods for the segmentation of pole-like features is that the points belonging to coaxial but spatially disconnected pole-like features will be segmented into the same cluster. In order to resolve this ambiguity, a grouping procedure is performed while considering the estimated local point density indices to discriminate these features in the spatial domain.

\subsection{Spatial-domain segmentation of pole-like features}

In this section, the proposed region growing technique for the segmentation and isolation of the detected pole-like features is introduced. This segmentation procedure starts by defining the points which potentially belong to pole-like features (i.e., those classified through the PCA procedure) as seed points. Afterwards, a spherical neighbourhood of each seed point is defined while considering the estimated local point density indices. The normal distances between the points within the established 3D neighbourhood and the surface of the pole-like feature associated with the seed points are then examined to check whether they are within the noise level in the TLS point cloud. The points which satisfy this condition are considered to be a part of the pole-like feature passing through the seed points.

In the next step, the aforementioned neighbouring points are used as seed points and the growing procedure continues until no additional laser points can be explored to belong to this polelike feature. The descriptor parameters of the segmented polelike features are sequentially refined using a least-squares cylinder fitting during the region growing procedure. In order to avoid growing pole-like features into the points which belong to planar features, a planar feature segmentation procedure should be initially implemented to detect and isolate planar surfaces. Figure 7 shows the region-growing procedure for the segmentation of pole-like features, graphically.

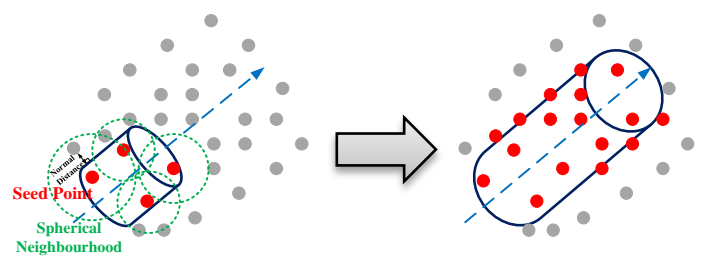

Figure 7. The region-growing procedure for the segmentation of pole-like features

\section{REGISTERATION OF MULTIPLE TLS SCANS}

Due to their perspective scanning mechanism, TLS systems are not usually able to provide a complete coverage of different objects in a single scan. This problem is also intensified by significant local point density variations throughout a single scan or occlusions caused by the other objects. Hence, multiple TLS scans from different viewpoints should be integrated to ensure a complete coverage of target objects in a scene. In order to integrate TLS scans properly, a registration procedure is performed to align these scans to a common reference frame. In this paper, the Iterative Closest Projected Point (ICPP) registration proposed by Al-Durgham et al. (2011) is utilized for the alignment of TLS scans.

This registration approach is implemented as follows: In the first step, a point to triangular patch (i.e., closest three points) match is established between overlapping TLS scans by checking if the point falls within a triangular dipyramid. This dipyramid is generated by extruding two points from the centroid of the aforementioned three neighbouring points. Then, the point is projected onto the patch surface, and its projection is used as a match for the original point. An appropriate mathematical model is then used to establish the relationship between a point and its projection. In our case, a threedimensional similarity transformation is used to represent the relationship between the involved scans. Figure 8 represents the implemented ICPP procedure for the registration of two TLS scans.

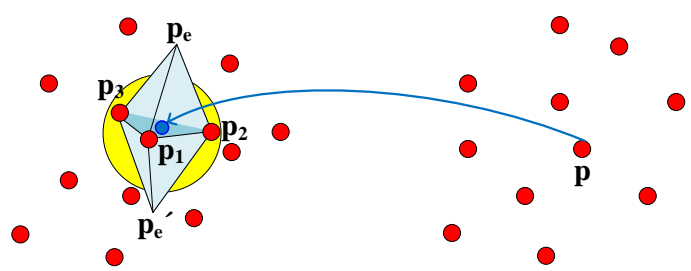

TLS scan 1

TLS scan 2

Figure 8. The representation of ICPP registration procedure

\section{QUALITY CONTROL OF POLE-LIKE FEATURES' SEGMENTATION RESULTS}

In order to evaluate the quality of the spatial-domain and parameter-domain pole-like feature segmentation outcome using single and multiple scans, a novel quality control procedure is introduced in this paper. This procedure addresses different issues affecting the quality of the segmentation results and establishes quantitative measures that give an indication about the frequency of such problems. As the first step of this procedure, the roughness factor of the pole-like features extracted using the proposed segmentation approaches is estimated. For each of the derived segments, the surface roughness factor is computed by estimating the Root Mean Square Error (RMSE) of the normal distances between the points within that segment and the surface of their best-fitted cylinder, as follows:

$$
\text { Surface Roughness Factor }=\sqrt{\frac{\sum_{i=1}^{n} n d_{i}^{2}}{n}}
$$

Where $n d_{i}$ is the normal distance between point $i$ (which belongs to a segmented pole-like feature) and the surface of the best-fitted cylinder through the entire cluster's points and $n$ is the number of the points aggregated in that segmented pole-like feature. In the following subsections, potential problems affecting the quality of the pole-like features' segmentation results are outlined and their pertinent quality control measures are defined. 


\section{Over-segmentation}

This problem occurs when a pole-like feature has been segmented into several pole-like features. This might happen due to problems either in the attribute computation or cluster detection process in parameter-domain or improper selection of seed regions in spatial-domain segmentation approaches. To check instances of this problem, we check if neighbouring segmented pole-like features, which have similar directional parameters, can be merged together as one pole-like feature. Two neighbouring pole-like features will be merged if the derived surface roughness factor for the merged segment is not more than a predefined threshold (e.g., 2 times of the larger segment's surface roughness factor). Once all neighbouring segments are checked for over-segmentation problem, the associated quality control measure can be derived according to Equation 3.

$$
\mathrm{QC}_{-} \text {measure }{ }_{1}=\frac{\mathrm{m}_{\mathrm{OS}}}{\mathrm{n}_{\mathrm{S}}}
$$

Where $n_{S}$ is the total number of initially segmented pole-like features and $m_{O S}$ is the number of pole-like features that can be merged into other segmented pole-like features as a result of the proposed quality control procedure.

\section{Under-segmentation}

This problem occurs when several pole-like features are segmented as one pole-like feature. In parameter-domain segmentation approaches, it might happen due to problems either in the attribute computation or cluster detection steps. In spatial-domain segmentation approaches, this problem might occur due to improper seed regions selection. In order to detect instances of this problem, the Average Surface Roughness Factor (ASRF) for all segmented pole-like features is estimated as in Equation 4.

$$
\text { ASRF }=\sum_{i=1}^{N} \frac{n_{i} . \text { Surface roughness } \text { factor }_{i}}{\sum_{i=1}^{N} n_{i}}
$$

Where $n_{i}$ is the number of points in the $i^{\text {th }}$ segment and $\sum_{i=1}^{N} n_{i}$ is the total number of the segmented points along the pole-like features. For each of the segmented pole-like features, if its surface roughness factor is significantly larger than the estimated average surface roughness factor, that pole-like feature is suspected to be under-segmented. Once all the segmented pole-like features are checked for the possibility of the under-segmentation problem occurrence, the associated quality control measure is derived as in Equation 5.

$$
\mathrm{QC}_{-} \text {measure } 2=\frac{\mathrm{m}_{\mathrm{US}}}{\mathrm{n}_{\mathrm{S}}}
$$

Where $n_{S}$ is the total number of the segmented pole-like features and $m_{U S}$ is the number of the segmented pole-like features that are suspected to be under-segmented. The suspected undersegmented pole-like features can be either re-segmented individually or checked with ground truth data for the possibility of separating them into multiple pole-like features.

\section{EXPERIMENTAL RESULTS}

In order to evaluate the performance of the proposed approaches for the segmentation of pole-like features while using a single or a multiple-registered TLS scans, experiments using real datasets are conducted in this section. These datasets include single and registered multiple TLS scans - five overlapping TLS scans aligned to a common reference frame using the ICPP registration technique - which have been acquired in an electrical substation using a FARO Focus ${ }^{3 \mathrm{D}}$ terrestrial laser scanner. Figures 9.a and 9.b show the original single and multiple-registered TLS scans, respectively.

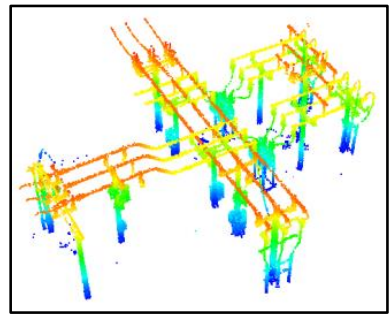

(a)

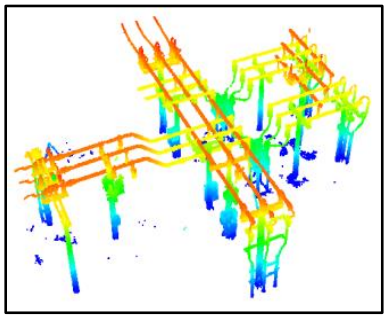

(b)
Figure 9. The original TLS point cloud: (a) single scan and (b) multiple-registered scans

These datasets are then processed using the proposed parameterdomain and spatial-domain segmentation techniques for the extraction of pole-like features. Figures 10.a and 10.b show the pole-like feature segmentation results using the proposed parameter-domain technique and Figures 11.a and 11.b show the segmentation results using the proposed spatial-domain approach, respectively.

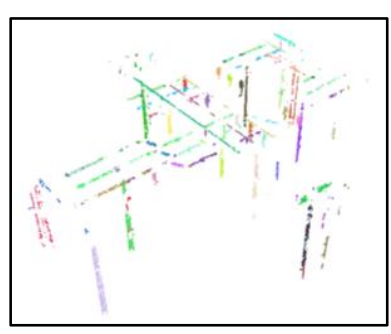

(a)

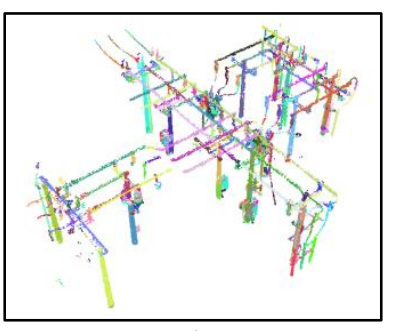

(b)
Figure 10. Parameter-domain pole-like feature segmentation results: (a) from a single TLS scan and (b) from multiple registered TLS scans

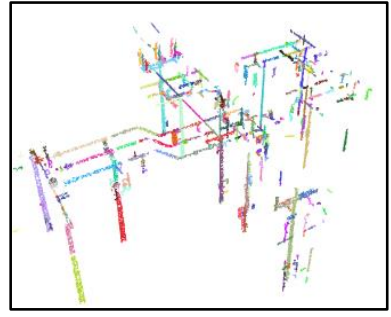

(a)

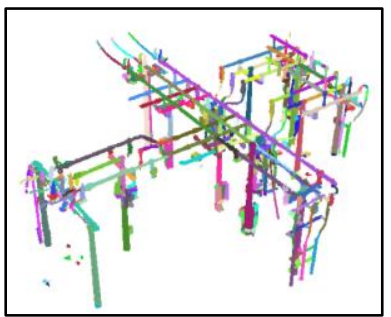

(b)
Figure 11. Spatial-domain pole-like feature segmentation results: (a) from a single TLS scan and (b) from multiple registered TLS scans

Qualitative evaluation of the derived segmentation results, through visual inspection of Figures 10 and 11, shows that the pole-like features have been segmented and extracted more completely using multiple-registered TLS scans. However, the single-scan segmentation results show the possibility of using the extracted features from the individual scans for the rough/coarse alignment of the scans to a common reference frame. The cross-sectional views of three segmented pole-like features from both single TLS scan and multiple-registered TLS scans, illustrated in Figure 12, confirm this conclusion. Moreover, this evaluation verifies that the proposed spatialdomain approach is able to extract more complete pole-like features due to its ability in considering the entire point cloud during the region growing procedure. The number/percentage of the segmented points along pole-like feature from single and registered TLS scans using the parameter-domain and spatial- 
domain segmentation techniques, listed in Table 1, also verifies this conclusion.

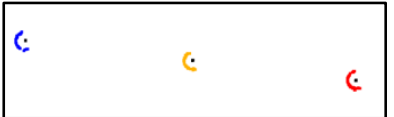

(a)

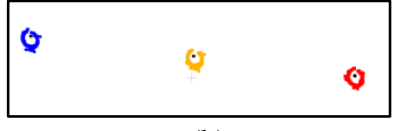

(b)
Figure 12. The cross-sectional view of three segmented polelike features: (a) from a single scan and (b) from multipleregistered scans

\begin{tabular}{|c|c|c|}
\hline \multirow{2}{*}{} & \multicolumn{2}{|c|}{$\begin{array}{c}\text { Number/percentage of the } \\
\text { segmented points along pole-like } \\
\text { features }\end{array}$} \\
\cline { 2 - 3 } & $\begin{array}{c}\text { Parameter-domain } \\
\text { Segmentation }\end{array}$ & $\begin{array}{c}\text { Spatial-domain } \\
\text { Segmentation }\end{array}$ \\
\hline Single TLS scan & $28,304 / 70 \%$ & $33,295 / 81 \%$ \\
\hline Registered TLS scan & $152,958 / 75 \%$ & $189,400 / 92 \%$ \\
\hline
\end{tabular}

Table 1. The number/percentage of segmented points along pole-like features using the proposed parameter-domain and spatial-domain segmentation approaches

The quantitative evaluation of the segmentation results is then performed using the proposed quality control procedure in the previous section. Table 2 summarizes the quality control measures of the segmentation results derived from the TLS datasets using the proposed parameter-domain and spatial domain segmentation approaches.

Analysis of these quality control measures shows that fewer instances of over-segmentation problem have been detected in the parameter-domain segmentation results. This probably happens due to underestimation of the utilized normal distance thresholds for growing some of the pole-like features. On the other hand, the frequency of the under-segmentation problem is higher in the parameter-domain segmentation results. This may happen due to problems either in attributes computation or cluster detection procedures. It can also be concluded that higher quality segmentation results have been derived for the registered TLS scan using both of the proposed segmentation approaches.

\begin{tabular}{|c|c|c|c|c|}
\hline \multirow{2}{*}{} & \multicolumn{2}{|c|}{$\begin{array}{c}\text { Parameter-domain } \\
\text { segmentation }\end{array}$} & \multicolumn{2}{c|}{$\begin{array}{c}\text { Spatial-domain } \\
\text { segmentation }\end{array}$} \\
\cline { 2 - 5 } & $\begin{array}{c}\text { Single } \\
\text { scan }\end{array}$ & $\begin{array}{c}\text { Registered } \\
\text { scans }\end{array}$ & $\begin{array}{c}\text { Single } \\
\text { scan }\end{array}$ & $\begin{array}{c}\text { Registered } \\
\text { scans }\end{array}$ \\
\hline $\begin{array}{c}\text { Over- } \\
\text { segmentation }\end{array}$ & $12 \%$ & $4 \%$ & $18 \%$ & $8 \%$ \\
\hline $\begin{array}{c}\text { Under- } \\
\text { segmentation }\end{array}$ & $5 \%$ & $0 \%$ & $1 \%$ & $0 \%$ \\
\hline
\end{tabular}

Table 2. Derived quality control measures for the segmented pole-like features from the utilized TLS datasets using the proposed segmentation approaches

\section{CONCLUSIONS AND RECOMMENDATIONS FOR FUTURE RESEARCH WORK}

This paper presented two recently-developed parameter-domain and spatial-domain approaches for the segmentation of pole-like features from TLS data. These approaches are implemented in three successive steps: In the first step, the points which potentially belong to pole-like features are detected and represented using a PCA procedure. In the second step, the local point density variations along the detected pole-like features are quantified and utilized for the precise computation of pole-like feature directional and positional parameters. In the third step, a novel parameter-domain clustering or region-growing procedure is performed for the segmentation and extraction of individual pole-like features. These approaches overcome the shortcomings of previously-developed approaches for the segmentation pole-like features by:

- Quantitative estimation and effective consideration of local point density variations during pole-like feature segmentation,

- Consideration of the data noise level in the pole-like feature segmentation process, and

- Implementation of computationally efficient procedures for aggregating the detected pole-like features in the parameter-domain or spatial-domain.

The complete segmentation and extraction of pole-like features from a single TLS using the proposed segmentation approaches is usually not feasible due to significant point density variations in the data and occlusions caused by other objects However, the segmentation of pole-like features from the individual scans can be used for the rough registration/alignment of neighbouring scans. In order to be able to extract the pole-like features more completely, the ICPP registration procedure was reviewed and utilized in this research for the alignment of multiple TLS scans collected in the same scene. The performance of the proposed parameterdomain and spatial-domain approaches for the segmentation of pole-like features was then investigated while using a single and multiple-registered TLS scans. Qualitative and quantitative evaluations of the derived segmentation results verify that more complete pole-like features can be extracted from registered TLS scans. They also confirm that the registration of multiple TLS scans from the same scene facilitates higher quality extraction and 3D modelling of objects of interest.

\section{ACKNOWLEDGEMENT}

The authors would like to thank TECTERRA for the financial support of this research work.

\section{REFERENCES}

Al-Durgham, M., Detchev, I., Habib, A., 2011. Analysis of two triangle-based multi-surface registration algorithms of irregular point clouds. in: The International Archives of the Photogrammetry, Remote Sensing and Spatial Information Sciences, part 5/W12, pp.61-66.

Belton, D., Lichti, D.D., 2006. Classification and segmentation of terrestrial laser scanner point clouds using local variance information. in: The International Archives of the Photogrammetry, Remote Sensing and Spatial Information Sciences, Part 5, pp. 44-49.

Bolles, R.C., Fischler, M.A., 1981. A RANSAC-based approach to model fitting and its application to finding cylinders in range data. in: Proceedings of the $7^{\text {th }}$ International Joint Conference on Artificial Intelligence - IJCAI'81, San Francisco, CA, USA, pp. 637-643.

Chaperon, T., Goulette, F., 2001. Extracting cylinders in full 3D data using a random sampling method and the gaussian image. in: Proceedings of the Vision Modeling and Visualization Conference 2001, VMV '01, pp. 35-42.

El-Halawany, S., Lichti, D.D., 2011. Detection of road poles from mobile terrestrial laser scanner point cloud. in: Proceedings of 2011 International Workshop on Multi-Platform/Multi-Sensor Remote Sensing and Mapping (M2RSM), pp. 1-6.

Fischler, M.A., Bolles, R.C., 1981. Random Sample Consensus: A paradigm for model fitting with applications to 
image analysis and automated cartography. Commun ACM, 24, pp. 381-395.

Friedman, J.H., Bentley, J.L., Finkel, R.A., 1977. An algorithm for finding best matches in logarithmic expected time. ACM Trans Math Softw, 3, pp. 209-226.

Gross, H., Thoennessen, U., 2006. Extraction of lines from laser point clouds. in: The International Archives of the Photogrammetry, Remote Sensing and Spatial Information Sciences, Part 3, pp. 86-91.

Kimme, C., Ballard, D., Sklansky, J., 1975. Finding circles by an array of accumulators. Commun ACM, 18, pp. $120-122$.

Lari, Z., Habib, A., 2013. New approaches for estimating the local point density and its impact on LiDAR data segmentation. Photogramm. Eng. Remote Sens, 79, pp. 195-207.

Lari, Z., Habib, A., 2014. An adaptive approach for the segmentation and extraction of planar and linear/cylindrical features from laser scanning data. ISPRS J. Photogramm. Remote Sens. doi:http://dx.doi.org/10.1016/j.isprsjprs.2013.12.001

Lukács, G., Martin, R., Marshall, D., 1998. Faithful leastsquares fitting of spheres, cylinders, cones and tori for reliable segmentation. in: proceedings of Computer Vision - ECCV'98, pp. 671-686.

Marshall, D., Lukacs, G., Martin, R., 2001. Robust segmentation of primitives from range data in the presence of geometric degeneracy. IEEE Trans. Pattern Anal. Mach. Intell., 23, pp. 304-314.

Pauly, M., Gross, M., Kobbelt, L.P., 2002. Efficient simplification of point-sampled surfaces. in: Proceedings of the Conference on Visualization '02, VIS '02. IEEE Computer Society, Washington, DC, USA, pp. 163-170.

Rabbani, T., 2006. Automatic reconstruction of industrial installations using point clouds and images. TU Delft: Civil Engineering and Geosciences.

Rabbani, T., van den Heuvel, F., 2005. Efficient Hough transform for automatic detection of cylinders in point clouds. in: The International Archives of the Photogrammetry, Remote Sensing and Spatial Information Sciences, part 3/W19, pp. 60-65.

Schnabel, R., Wahl, R., Klein, R., 2007. Efficient RANSAC for point-cloud shape detection. Comput. Graph. Forum, 26, pp. 214-226.

Vosselman, G., Gorte, B.G.H., Sithole, G., Rabbani, T., 2004. Recognising structure in laser scanner point clouds. in: The International Archives of the Photogrammetry, Remote Sensing and Spatial Information Sciences, part 8/W2, pp. 33-38. 